A LINIT PKOOF OF THE THEOREM EUC. III, 35 .

are conjugate, for the polar of either passes through the other. Similarly if $P \mathbf{2}$ and $P 5$ are at right angles, 2,5 are conjugate. Hence 3,6 are conjugate, and $P 3, P 6$ ate at right angles, i.e The circles on the three diagonals as diameters are coaxial.

(d) Take for the conic the pair of lines through any point $Q$, harmonically conjugate both to $Q 1, Q 4$ and to $Q 2, Q 5$. Then $Q 3, Q 6$ are also harmonically conjugate to the same pair of lines, $i$ e. The lines from any point to the six vertices are in involution.

The dual of this is: The three pairs of opposite sides of a quadrangle are cut in involution by any transversal.

John Dougall.

\title{
A Limit Proof of the Theorem Euc. III, 35.
}
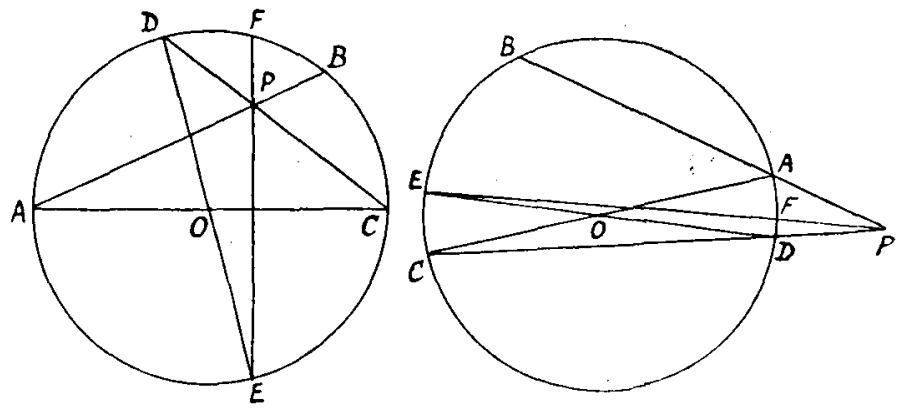

Let $A B$ be any chord passing through the fixed point $P$. Draw the diameter $A O C$, join $C P$ and let it meet the circumference at $D$. Then in triangle $A C P, A C^{2}=A P^{2}+C P^{2}$ together with either $2 A P . P B$ or $2 C P$. $P D$ according as $C P$ is projected on $A P$ or $A P$ on $C P$. Hence $A P . P B=C P . P D$. Now draw the diameter $D O E$, join $E P$ and let it meet the circumference at $F$. Then the theorem is true for chords $C D, E F$. 
By repeating indefinitely this process we arrive at the fixed diameter through $P$ as the limiting chord. Hence the theorem is true generally.

The proof is applicable unchanged to the case of secants if the sign convention is admitted, and with simple modification if it is not.

G. D. C. Stokes.

\section{On Recurring Decimals.}

$\S 1$. The present note gives two methods by which vulgar fractions may often be converted into decimals with great 1 apidity. It is not pretended, however, that they are more than mere curiosities or that they are of practical use. The first method is not new, but $I$ have never seen the secoud mentioned anywhere. The proofs are almost self-evident.

$\$ 2$. If the vulgar fraction have its numerator and denominatur multiplied, if necessary, by the same factor so that the significant digits of the denominator become $m \cdot 10^{n}-1$, where $m$ is an integer so small that we can perform short division by it mentally, then the complete recurring period can be written straight down with ease. The best description will be provided by an example.

Ex. To convert into a recurring decimal $\frac{53}{8}$.

We have $87 \times 45977=3999999=4 \times 10^{6}-1$,

$$
53 \times 45977=2436781 \text {. }
$$

(It will be shown later how we find the multiplier 45977.)

$$
\therefore \quad \frac{53}{87}=\frac{2436781}{3999999} \text {. }
$$

Write down the numerator, 2436781 , and divide it by 4 . When six digits have been obtained (six, because of the $10^{6}$ ), transfer the quotient to a new row just below, continuing division of the row just quitted, "carrying" of course any remainder from the first row. When this new row of the quotient has six digits begin 